\title{
A Correlative Imaging Approach for Extracellular Matrix Characterization in Mice
}

Yifei Liu ${ }^{*}$, Yong-Ung Lee ${ }^{2}$, Tai $\mathrm{Yi}^{2}$, Ken Wu${ }^{3}$, Cedric Bouchet-Marquis ${ }^{3}$, Han Chan ${ }^{3}$, Christopher K. Breuer $^{1,2}$, David W. McComb ${ }^{1}$

1. Center for Electron Microscopy and Analysis, The Ohio State University, Columbus, OH, USA

2. Nationwide Children's Hospital, Center for Regenerative Medicine, Columbus, OH, USA

3. Thermo Fisher Scientific, Hillsboro, OR, United States

* Corresponding author: liu.612@osu.edu

The pulmonary valve is responsible for providing unidirectional flow or deoxygenated blood from the right ventricle to the lungs in mammals. In a normal cardiac cycle, the pulmonary valve opens at high ventricular pressures (systole) and closes during muscle relaxation (diastole). As a pure biomechanical structure, its mechanical behavior can completely be explained by extracellular matrix (ECM) characterization. It has been hypothesized that ECM proteins undergo reorganization in order to accommodate the large amounts of strain [1]. One such ECM protein is collagen, which is responsible for the mechanical strength of the valve [2]. Valvular biomechanics has been extensively studied in many animal models, but very little work has been done regarding the murine model. The mouse model is advantageous because of the ability to produce large identical populations, but the knowledge gap is due to the surgical and instrumental capabilities necessary to adequately characterize the extracellular matrix. Histology and optical microscopy have been the main methods responsible for the current understanding of murine pulmonary valve architecture, but accurate comparison across multiple specimens have proven difficult due to information lost during sample processing [3,4]. Firstly, the structure of the pulmonary valve consists of three individual leaflets, each structurally and temporally heterogeneous. In order to reproducibly compare ECM architecture, the conformation of the leaflet needs to be controlled. Secondly, the pulmonary trunk is roughly $1 \mathrm{~mm}$ in height, the leaflets can range from 10-200 um in thickness and the collagen fibers are on the order of nanometers. Imaging across this large length spectrum cannot be accomplished by a single method. Furthermore, correlation of images across multiple imaging modalities is essential to account for the structural variance. We propose a correlative workflow utilizing microcomputed tomography $(\mu \mathrm{CT})$, scanning electron microscopy (SEM) in combination with serial block face imaging (SBFI) and transmission electron microscopy (TEM) to capture the three-dimensional collagen, an ECM component, organization within murine heart valve.

Wild-type Black 6 mice were anesthetized with a solution of ketamine/xylazine in accordance to IACUC protocols. Systemic blood was flushed out and the heart was excised. The pulmonary valve was closed by arterial hydrostatic pressure. The conformation of the leaflets was fixed using a solution glutaraldehyde/paraformaldehyde in cacodylate buffer. The pulmonary valve was isolated and stained using osmium tetroxide, uranyl acetate and lead aspartate. Specimens were then dehydrated by successive treatments of ethanol and embedded in resin.

Sample excision and processing make retainment of orientation difficult. In order to reobtain this information, embedded samples were scanned and reconstructed using a Heliscan $\mu$ CT (Thermo Fisher Scientific) with a voxel size of $5.4 \mu \mathrm{m}$. From $\mu \mathrm{CT}$ volume renderings, we can precisely determine the conformation of the pulmonary valve. Fig. 1A shows an example volume rendering from Avizo image processing and data analysis software (Thermo Fisher Scientific) and a virtual slice along the major axis 
of the pulmonary artery, Fig 1B. With guidance from the $\mu \mathrm{CT}$ volume rendering, we manipulated our sample in a microtome to precisely slice towards a region of interest. A voxel size of $5.4 \mu \mathrm{m}$ from the $\mu \mathrm{CT}$ is only sufficient to resolve the gross conformation of the pulmonary valve. In order to resolve collagen, specimens were imaged under an Apreo Volumescope SEM (Thermo Fisher Scientific) equipped with SBFI capability for automated image stack acquisition. A Volumescope is an SEM equipped with an in-chamber microtome using a diamond knife (Diatome) to serial section embedded samples for slice and view applications. Fig 1B-C shows an example of the visual correlation between the $\mu \mathrm{CT}$ virtual slice to the SEM image. Multiple regions of interest an be acquired simultaneously using different field of views and resolutions during the SBFI process. The resulting stack of images were reconstructed into a volume rendering of the pulmonary valve that resolves the ECM. Fig 2 shows an overview image with a pixel size of $146 \mathrm{~nm}$ and subregion imaged with pixel size of $10 \mathrm{~nm}$. This data set now provides insight into the orientation of collagen and can help elucidate the resulting biomechanics.

\section{References:}

[1] MS Sacks, et al. Journal of Biomedical Materials Research 41 (1998), p. 131.

[2] P Fratzl., et al. Journal of structural biology 122 (1998), p. 119.

[3] RB Hinton, et al. Circulation research (2006), p. 1431.

[4] RB Hinton and KE Yutzey. Annual Review of Physiology 73 (2011), p. 29.

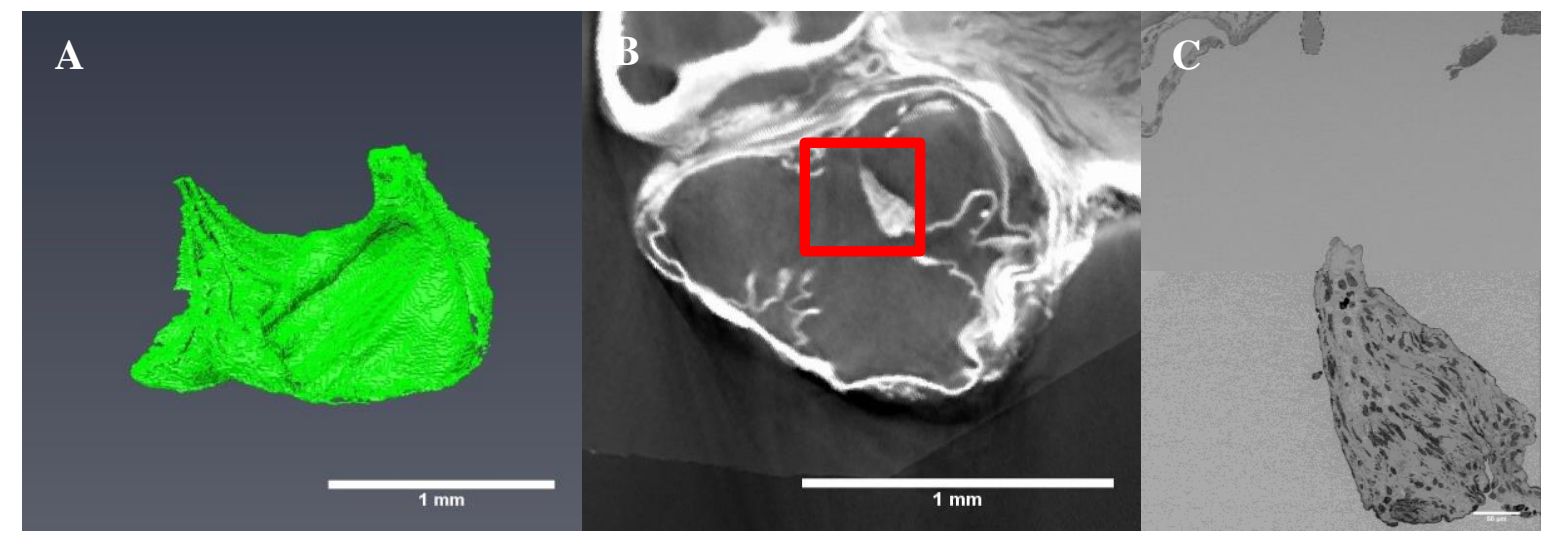

Figure 1: (A) $\mu \mathrm{CT}$ volume rendering of pulmonary valve fixed under physiological pressure with glutaraldehyde/paraformaldehyde. (B) Virtual slice of $\mu \mathrm{CT}$ along major axis of pulmonary artery. Red box in (B) indicates cross-section imaged by SEM in (C).

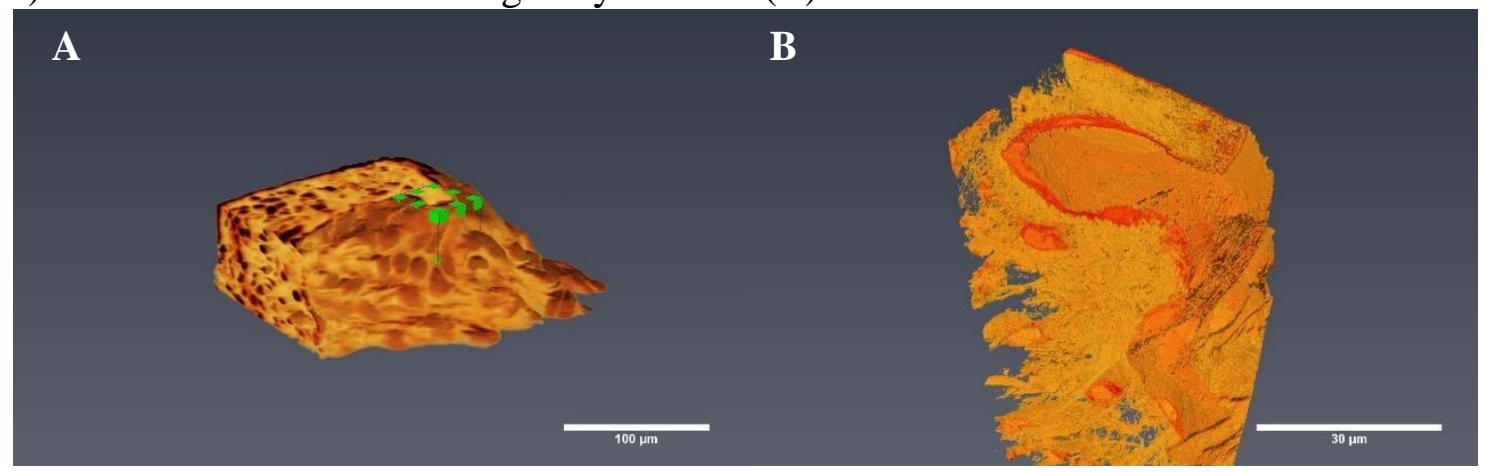

Figure 2: Volumescope volume rendering of (A) overview images with pixel size of $146 \mathrm{~nm}$ and (B) region of interest images with pixel size of $10 \mathrm{~nm}$. Green rectangular region corresponds in (A) corresponds to region of (B). 